\title{
Exercise training improves cardiac function in infarcted rabbits: involvement of autophagic function and fatty acid utilization
}

\author{
Ching-Yi Chen ${ }^{1}$, Hsiu-Ching Hsu ${ }^{2}$, Bai-Chin Lee ${ }^{2}$, Hung-Ju Lin ${ }^{2}$, Ying-Hsien Chen ${ }^{2}$, \\ Hui-Chun Huang ${ }^{2}$, Yi-Lwun Ho ${ }^{2}$, and Ming-Fong Chen ${ }^{2 *}$
}

${ }^{1}$ Department of Animal Science and Technology, National Taiwan University, 50 Lane 155, Sec. 3, Keelung Rd., Taipei, Taiwan; and ${ }^{2}$ Department of Internal Medicine, National Taiwan University Hospital, 7 Chung-Shan South Road, Taipei, Taiwan

Received 9 September 2009; revised 4 December 2009; accepted 17 December 2009

\begin{abstract}
Aims
To explore whether exercise can improve cardiac function in a post-myocardial infarction (MI) rabbit model and to determine contributing factors in the left ventricle (LV).

Methods and results

Adult male New Zealand White rabbits $(2.5-3 \mathrm{~kg})$ underwent $\mathrm{Ml}$ by ligation of the left anterior descending coronary artery. For 8 weeks after surgery, sham-operated, and post-Ml rabbits were housed under sedentary conditions or assigned to a 4-week treadmill exercise protocol at a speed of $1.0 \mathrm{~km} / \mathrm{h}$ for $30 \mathrm{~min} 5$ days per week, then sacrificed. The non-infarcted region of the LV was harvested for further analysis. MI decreased left ventricular ejection fraction (LVEF) and increased thiobarbituric acid reactive substances (TBARS) generation in the LV. Exercise improved the cardiac function of MI rabbits. Left ventricular LC3II/LC3I (microtubule-associated protein light chain 3) in the MI group was 2.1-fold higher than that of the sham group, exercise significantly decreased LC3II/LC3I in the MI group. MI down-regulated the expression of heart-type fatty acid binding protein (h-FABP), and exercise up-regulated h-FABP. In addition, LVEF had a significantly positive correlation with h-FABP and a negative correlation with LC3II/ LC3I.
\end{abstract}

Conclusion Exercise induced change in autophagic function and fatty acid utilization may contribute to the improvement in ventricular function in the infarcted heart.

Keywords Exercise training $\bullet$ Myocardial infarction $\bullet$ Autophagic function $\bullet$ Fatty acid utilization $\bullet$ Oxidative status

\section{Introduction}

Myocardial infarction (MI) is one of the most important aetiologies of heart failure. Cardiac remodelling after $\mathrm{Ml}$ consists of a series of changes in the left ventricle (LV), leading to a decline in LV performance. ${ }^{1-4}$ Importantly, severe $\mathrm{Ml}$ can result in permanent disability or death. Recently, exercise has become a potential therapy for attenuating LV dysfunction. Numerous putative mechanisms of exercise-induced cardioprotection against myocardial injury have been proposed and investigated. These mechanisms include anatomical changes in the coronary arteries, induction of myocardial heat shock proteins, an increase in myocardial cyclooxygenase-2 activity, elevation of endoplasmic reticulum stress proteins, improvement of function of sarcolemmal ATP-sensitive potassium channels and mitochondrial ATP-sensitive potassium channels, improvement of insulin resistance, promotion of angiogenesis, and attenuation of LV remodelling in the post-MI failing heart. ${ }^{2-4}$

Exercise improves skeletal muscle lipid oxidation and plasma antioxidant enzyme activity in animals with chronic heart failure. $^{5-8}$ Increasing the antioxidant enzyme activity of glutathione peroxidase and superoxide dismutase in the failing heart reduces cardiac injury. ${ }^{9,10}$ Since exercise induces higher myocardial oxygen demands ${ }^{11,12}$ and the failing heart has a limited ability for superoxide removal, ${ }^{1}$ an important issue for exercise therapy is whether antioxidant capacity is enough to conquer the oxidative stress of the failing heart.

* Corresponding author. Tel: +886 22312 3456, Fax: +886 23322 3937, Email: mfchen@ntu.edu.tw 
Programmed cell death (PCD) is classified into typical apoptosis (PCD-1) and autophagic cell death (PCD-2), which differ in the predominance of caspase activation or autophagy, respectively. ${ }^{13,14}$ Both types of PCD have been observed in cardiac myocytes. Morphologically, PCD-1 is characterized by chromatin condensation and the fragmentation of the nucleus and cytoplasm into apoptotic bodies, although PCD-2 is associated with cell shrinkage and the formation of multiple autophagic vacuoles. ${ }^{13,14}$ In addition to its role in PCD-2, autophagy functions in the recycling of cytoplasmic organelles and proteins at low basal levels under normal conditions and is up-regulated in response to stresses such as ischaemia/ reperfusion (I/R) and in cardiovascular diseases such as heart failure. $^{15-17}$ Autophagy recycles the cytoplasmic portions in double-membrane sequestering vesicles called autophagosomes. ${ }^{17}$ Microtubule-associated protein light chain 3 (LC3), which is the main substrate for the autophagosome membrane, is synthesized and then cleaved at glycine 120 to form the cytosolic LC3I. Upon induction of autophagy, the C-terminal glycine of LC3I is conjugated with phosphatidylethanolamine and is presented in isolation on membranes of the autophagosomes as LC3II. ${ }^{18}$ The autophagosome is delivered to the lysosome compartments where it is broken down into its essential constituents and recycled back into the cytoplasm. Impairment of autophagic function causes protein to aggregate and accumulate as autophagosomes in the cytoplasm, leading to cell death. ${ }^{17}$

There is increasing evidence to show that exercise up-regulates autophagy, and increases the protein turnover rate; ${ }^{19-21}$ however, it is unknown whether exercise has the same effect on the failing heart. In the present study, we investigated the effect of exercise training on cardiac function in an infarcted rabbit model explored the possible mechanisms of this effect, by analysing biomarkers of oxidative stress, antioxidant capacity, autophagy, apoptosis, and fatty acid utilization.

\section{Methods}

\section{Animal model and experimental protocol}

Male New Zealand White rabbits weighing $2.5-3 \mathrm{~kg}$ were subjected to $\mathrm{Ml}$ by ligation of the left anterior descending coronary artery as previous described. ${ }^{22}$ One month later, the rabbits were randomly allocated to two groups: (i) exercise rabbits assigned to a 4-week training programme and (ii) sedentary rabbits confined to their cages during the same time period. Exercise training was performed on a low-speed, levelled, motorized treadmill (model LDM300D, Lee Dah Mei Industry Co, Ltd., Taiwan). The training programme was preceded by a 2-week period of adaptation to the treadmill exercise, during which the running time and speed of the treadmill were gradually increased from $10 \mathrm{~min}$ at $0.5 \mathrm{~km} / \mathrm{h}$ to $30 \mathrm{~min}$ at $1 \mathrm{~km} / \mathrm{h}$. Exercise training consisted of a 4-week period of running at a speed of $1 \mathrm{~km} / \mathrm{h}$ for 30 min 5 days per week. This exercise intensity and duration have been demonstrated to ameliorate endothelium-dependent vascular dysfunction and reduce pro-inflammatory changes in rabbits caused by a high-cholesterol diet. ${ }^{23}$

At the end of the study period, all the rabbits were anaesthetized and sacrificed with a pentothal overdose. The non-infarcted region of the LV (counter part of the infarcted area, remote zone) from each rabbit was cut into small pieces and stored in liquid nitrogen until analysis. All experiments were approved by the Institutional
Animal Care and Use Committee of National Taiwan University and were carried out in accordance with the National Institutes of Health (NIH Publication no. 85-23, revised 1996) and the American Physiological Society's Guide for the Care and Use of Laboratory Animals.

\section{Cardiac magnetic resonance imaging}

In order to measure LV volume, mass, function, and infarct size, animals were examined using a 3-T magnetic resonance imaging (MRI) unit (Trio; Siemens, Erlangen, Germany) with an eight-channel cardiac phased-array coil for signal reception. ${ }^{24}$ An ECG-gated turbo fast low angle shot (TurboFLASH) cine pulse sequence was acquired in two long-axis and five to seven short-axis views. After cine imaging was completed, an intravenous bolus dose of $0.20 \mathrm{mmol} / \mathrm{kg}$ of gadodiamide (Nycomed Imaging AS, Oslo, Norway) was administered, and late gadolinium enhancement images were acquired 10 min later using an inversion-recovery prepared segmented TurboFLASH sequence. LV volume, mass, and ejection fraction were assessed using cine MRI and an automated boundary detection algorithm. To assess infarct size, we quantified the late gadolinium enhancement using a signal intensity threshold criterion of $>2$ standard deviations above the mean signal intensity of the remote myocardium, and expressed it as a percentage of the total LV mass. Imaging analysis was performed using the Mathematica software package (Wolfram Research, Inc., IL, USA) and Matlab (MathWorks, Inc., Natick, MA, USA). The intra-observer variability in the LV volume results was $3 \%$.

\section{Oxygen radical absorbance capacity assay}

Total antioxidant capacity was analysed by the oxygen radical absorbance capacity (ORAC) assay, which is based on the oxidation of fluorescein by peroxyl radicals via a classic hydrogen atom transfer mechanism and provides a direct measurement of antioxidant capacity against hydrophilic chain-breaking peroxyl radicals. ${ }^{25}$ For analysis by the ORAC assay, the LV was pulverized under liquid nitrogen, weighed out $(50 \mathrm{mg})$ as frozen material and added to $1 \mathrm{~mL}$ of ice-cold $75 \mathrm{mM} \mathrm{K2HPO4/NaH2PO4} \mathrm{phosphate} \mathrm{buffer} \mathrm{(} \mathrm{pH}$ 7.0). The tissue samples were homogenized and centrifuged. The pellet was resuspended in homogenization buffer and centrifuged. The two supernatants were combined in a volumetric flask, adjusted to $2 \mathrm{~mL}$ of total volume, and used for the ORAC assay.

The ORAC assay was performed as described by Broke et al. ${ }^{25}$ with minor modifications. Measurements were performed on a Perkin Elmer Luminescence Spectrometer LS50 (Perkin Elmer, Waltham, MA, USA) with 542-nm excitation and 576-nm emission filters, conducted at $37^{\circ} \mathrm{C}$ in $75 \mathrm{mM}$ phosphate buffer ( $\mathrm{pH}$ 7.0). The fluorescence probe was phycoerythrin (PE) and the peroxyl radical generator was 2,2'-azobis (2-amidinopropane) dihydrochloride (AAPH). As a control standard, 6-hydroxy-2,5,7,8-tetramethylchroman-2-carboxylic acid (Trolox) was used. Each sample was analysed in duplicate using a 'forward-then reverse' ordering in each plate row. To each well of the preheated microplate, $30 \mu \mathrm{L}$ of sample (blank, phosphate buffer; Trolox, $1 \mathrm{M}$ final conc. or tissue extract) and $100 \mu \mathrm{L}$ PE $(3.78 \mathrm{mg} / \mathrm{L})$ were added, the plate was then shaken for $30 \mathrm{~s}$ and initial fluorescence was measured. AAPH solution ( $30 \mu \mathrm{L} ; 20 \mathrm{mM}$ final conc.) was added to each well, and after shaking the plate measurements were taken every 15 min until the fluorescence was $<5 \%$ of the initial reading. All fluorescence measurements were expressed relative to the initial reading. Results were calculated by using the differences of areas under the PE decay curves between the blank and each sample. The final results (ORAC values) were calculated by linear regression of the areas vs. sample concentration and expressed as mole Trolox equivalents (TE) per gram of myocardial tissue protein (mol TE/g). 


\section{Measurement of thiobarbituric acid reactive substances and 3-nitrotyrosine}

The LV samples were homogenized in lysis buffer $(0.25 \mathrm{M}$ sucrose, $1 \mathrm{mM}$ EDTA, $10 \mathrm{mM}$ Tris, $\mathrm{pH7.4}$ ) and the tissue homogenates were prepared for thiobarbituric acid reactive substances (TBARS) and 3nitrotyrosine (3-NT) analysis. TBARS were measured by fluorometric assay (excitation at $515 \mathrm{~nm}$, emission at $552 \mathrm{~nm}$ ) using 1,1,3,3-tetraethoxypropane as the standard, as described by Chen et al. ${ }^{26}$ The linearity $\left(R^{2}\right)$ of the standard curves was 0.991 . The coefficients of variation $(\mathrm{CV})$ of the intra- and inter-run from quality control samples were 5.02 and $6.98 \%$, respectively. The levels of total 3-NT was measured by a competitive enzyme-linked immunosorbent assay using a commercial kit according to the manufacturer's instructions (Millipore, Billerica, MA, USA). The intra- and inter-batch CV from quality control samples were 6.09 to $6.43 \%$, respectively. The amount of total nitrotyrosine in the LV tissue homogenates was estimated by comparing it with nitrated BSA as a standard.

\section{Determination of reduced (GSH) and oxidized (GSSG) glutathione levels in the non-infarcted part of the left ventricle}

The procedure for determining the total (GSH + GSSG) glutathione content in the non-infarcted part of the LV has been described previously. ${ }^{26}$ All procedures were performed at $30^{\circ} \mathrm{C}$. Twenty milligrams of tissue was lysed in $2 \mathrm{~mL}$ of $5 \%$ trichloroacetic acid, then $50 \mu \mathrm{L}$ of the lysate was added to $150 \mu \mathrm{L}$ of the glutathione assay mixture, which consisted of $0.6 \mathrm{nM}$ 5,59-dithiobis-(2-nitrobenzoic acid), $0.4 \cup$ of glutathione reductase, and $0.2 \mathrm{nM}$ reduced nicotinamide adenosine dinucleotide phosphate, the mixture was then incubated for $1.5 \mathrm{~min}$. The absorbance at $410 \mathrm{~nm}$ was then measured and the concentration calculated from a standard curve prepared using dilutions of a known GSSG or GSH concentration. For the GSSG assay, $\mathrm{N}$-ethylmaleimide $(0.02 \mathrm{~N}$ final concentration) was added to the lysate to remove GSH by forming a stable complex to prevent it from interfering with the assay. Following incubation for $60 \mathrm{~min}$ at $25^{\circ} \mathrm{C}$, the solution was extracted with 10 volumes of ether to ensure complete removal of the unreacted sulfhydryl reagent which would inhibit glutathione reductase activity in the assay mixture. After the extraction process, the solution was added to the assay mixture for GSSG determination. GSH levels were calculated by subtracting the GSSG value from the GSSG + GSH value.

\section{Western blotting}

Tissue samples were homogenized in RIPA lysis buffer (Santa Cruz, Santa Cruz, CA, USA), and protein concentrations were measured using the protein assay kit (Bio-Rad, Hercules, CA, USA). Protein suspension from the tissue lysate $(20 \mu \mathrm{g})$ was loaded onto a SDS-PAGE gel for electrophoresis and then transferred to a PVDF membrane (Amersham Bioscience). The membrane was then incubated for 1$2 \mathrm{~h}$ at room temperature with primary antibodies against $L C 3, B c 12$, Bax, heart-type fatty acid binding protein (h-FABP) and actin (Santa Cruz), respectively. After washing with PBS-T, the membrane was incubated with secondary antibody against goat or mouse lgG and the immunoblotting was visualized using QUANTITY ONE (Bio-Rad).

\section{Statistics}

All data are expressed as the mean \pm SD. Differences between groups were assessed by analysis of variance followed by a Tukey's test. Statistical analyses were performed using SAS (version 9.1; SAS Institute Inc., Cary, NC, USA). A P-value $<0.05$ was considered significant.

\section{Results}

A total of 40 rabbits underwent surgery, of these 26 were subjected to $\mathrm{Ml}$ and 14 to sham surgery. Of the rabbits with MI, six died within $24 \mathrm{~h}$ of the operation and four died after the operation (38.5\% post-operative mortality). The surviving 16 rabbits with $\mathrm{Ml}$ were either left untreated $(M, n=8)$ or assigned to an exercise training programme for 4 weeks (ME, $n=8$ ). Sham rabbits were assigned equally as untreated $(S, n=7)$ or treated with exercise training (SE, $n=7)$. There were no significant differences between the $M$ and $M E$ groups in terms of body weight, cardiac structure and function, or infarct size at 2 months after coronary ligation (data not shown). All rabbits survived until sacrificed three months post-MI. The non-infarcted region of the LV (counter part of the infarcted area, remote zone) from each rabbit was used to analyse biomarkers of oxidative stress, total antioxidant capacity, PCD, and fatty acid utilization.

\section{Cardiac function}

Left ventricular ejection fraction (LVEF) was used to evaluate cardiac function. No significant differences in cardiac function were observed between S and SE rabbits (LVEF for S and SE: $64.3 \pm 3.4$ and $66.0 \pm 3.9 \%$, respectively; Figure 1). The cardiac function of $\mathrm{Ml}$ rabbits had deteriorated 12 weeks after surgery; however, exercise training significantly increased the cardiac function of the MI rabbits (LVEF for M and ME: $40.1 \pm 3.6$ and $53.4 \pm$ $3.6 \%$, respectively; $P<0.05)$. These results indicate that $M I$ impaired the cardiac function of the rabbits and that exercise training improved the cardiac function of post-Ml rabbits.

\section{Oxidative status}

Total antioxidant capacity was analysed by ORAC assay (Table 1). Myocardial infarction tended to decrease left ventricular total antioxidant capacity but this difference was not significant. Exercise training has a different effect on sham and MI rabbits: it kept antioxidant capacity at a low level in the MI rabbits, although it

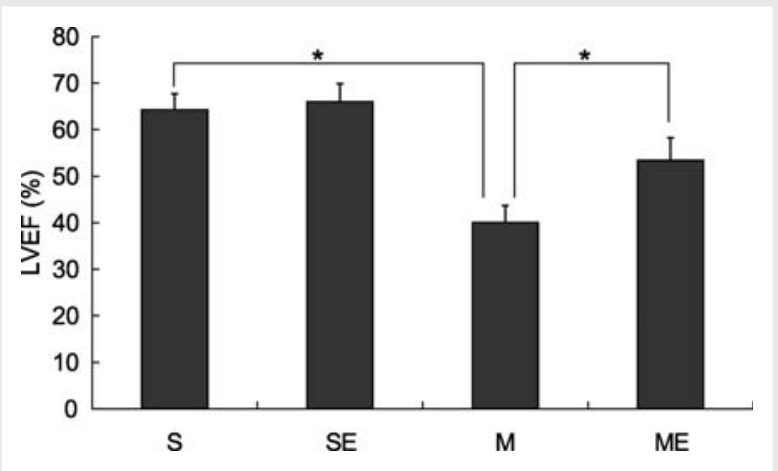

Figure I Effect of myocardial infarction and exercise training on left ventricular function of rabbit hearts. Data are means \pm SD (S and SE: $n=7$; M and ME: $n=8$, respectively; *, $P<0.05)$. S, adult rabbits with sham operation; $M$, adult rabbits with myocardial infarction for three months; $E$, rabbits assigned to a 4-week treadmill exercise protocol at a speed of $1.0 \mathrm{~km} / \mathrm{h}$ for 30 min 5 days per week. 
Table I Effect of myocardial infarction and exercise training on the antioxidative system in the rabbit left ventricle

\begin{tabular}{|c|c|c|c|c|}
\hline & $S(n=7)$ & SE $(n=7)$ & $M(n=8)$ & $\operatorname{ME}(n=8)$ \\
\hline Total antioxidant capacity ( $\mu$ M TE/mg protein) & $3594 \pm 189$ & $4091 \pm 219^{*}$ & $3244 \pm 242$ & $3060 \pm 212$ \\
\hline TBARS (nmol/mg protein) & $13.5 \pm 1.9$ & $14.2 \pm 2.2$ & $17.1 \pm 2.0 *$ & $18.4 \pm 2.2^{\S}$ \\
\hline 3-Nitrotyrosine ( $\mu$ g nitrated BSA/mg protein) & $2.61 \pm 0.44$ & $1.41 \pm 0.44 *$ & $1.36 \pm 0.47 *$ & $2.10 \pm 0.42$ \\
\hline GSH (ng/mg tissue) & $385.8 \pm 21.3$ & $427.4 \pm 22.7$ & $406.6 \pm 23.3$ & $422.1 \pm 27.8$ \\
\hline GSSG (ng/mg tissue) & $94.9 \pm 3.0$ & $94.3 \pm 3.9$ & $93.4 \pm 4.8$ & $91.9 \pm 3.2$ \\
\hline $\mathrm{GSH}+\mathrm{GSSG}$ (ng/mg tissue) & $480.7 \pm 22.7$ & $521.7 \pm 26.9$ & $500.0 \pm 24.8$ & $513.9 \pm 25.2$ \\
\hline
\end{tabular}

Data show the means \pm SD. TE, trolox equivalents; TBARS, thiobarbituric acid reactive substances; BSA, bovine serum albumin; GSH, reduced glutathione; GSSG, oxidized glutathione. S, adult rabbits with sham operation; $\mathrm{M}$, adult rabbits with myocardial infarction for 3 months; $\mathrm{E}$, rabbits assigned a 4-week treadmill exercise protocol at a speed of $1.0 \mathrm{~km} / \mathrm{h}$ for $30 \mathrm{~min} 5$ days per week.

$* P$-value for the $S$ vs. other group $<0.05$.

${ }^{\S} P$-value for the $S E$ vs. ME $<0.05$.

significantly elevated cardiac antioxidant capacity in the sham rabbits. TBARS was applied as the indicator of lipid peroxidation. Myocardial infarction caused a significant increase in cardiac TBARS levels (TBARS for $S$ and $M: 13.5 \pm 1.9$ and $17.1 \pm$ $2.0 \mathrm{nmol} / \mathrm{mg}$ protein, respectively). Exercise training did not affect cardiac TBARS production either in the $\mathrm{Ml}$ rabbits or in the sham rabbits. Tyrosine is a common target for nitrosative stress and 3-NT generation represents a marker for nitrosative stress. $^{27} \mathrm{Ml}$ rabbits had a lower level of cardiac 3-NT than did sham rabbits. Exercise training significantly decreased cardiac 3-NT generation in the sham group, whereas it tended to increase 3-NT generation in the Ml group but this difference was not significant. The cardiac levels of GSSG and GSH were not significantly different among the four groups. These results show lower antioxidant capacity and higher TBARS in the failing heart. Exercise training increased cardiac antioxidant capacity and attenuated nitrosative stress in the sham group, but not in the Ml group.

\section{Programmed cell death}

\section{Apoptosis}

$\mathrm{Bcl} 2$ and Bax were analysed as indicators of anti-apoptotic properties and pro-apoptotic properties, respectively. Twelve weeks after $\mathrm{Ml}$, cardiac $\mathrm{Bcl} 2$ expression was similar between the sham and $\mathrm{MI}$ groups (Figure $2 A$ and $B$ ). Exercise training slightly increased $\mathrm{Bcl} 2$ expression in the sham rabbits but this tendency did not reach a significant difference. In the $\mathrm{Ml}$ group, exercise training did not cause any differences in $\mathrm{Bcl} 2$ expression. A comparable pattern in cardiac Bax expression among treatments was observed. Bax expression in the LV was similar between the sham and $\mathrm{MI}$ groups. In the Ml rabbits, exercise training did not cause a significant change in Bax expression, whereas it significantly up-regulated Bax expression in the sham group. The up-regulation of Bax and slightly increased expression of $\mathrm{Bcl} 2$ in the sham rabbits by exercise training resulted in a lower ratio of $\mathrm{Bc} 22$ to $\mathrm{Bax}$, and therefore there were no differences between treatments. These results indicate that exercise training did not affect apoptosis either in the sham group or in the Ml group.

\section{Autophagy}

The amount of LC3-II and the ratio of LC3II to ILC3I are closely correlated with the number of autophagosomes, and serve as indicators of autophagosome formation. ${ }^{18}$ Myocardial infarction did not cause any change in left ventricular LC3I expression (Figure $2 \mathrm{~A}$ and $\mathrm{C}$ ). Exercise training significantly increased LC3I expression in the sham rabbits (1.6-fold), although it did not regulate LC3I expression in the MI rabbits, indicating that exercise training enhanced the autophagic function in the sham group, but not in the MI group. Cardiac LC3II expression was higher in the $\mathrm{Ml}$ group than in the sham group. Exercise training significantly decreased LC3II expression in the MI group; however, it tended to increase LC3II expression in the sham group but this difference was not significant. The MI group had a higher ratio of LC3II to LC3I than the sham group. Exercise training caused a lower ratio of LC3II to LC3I in the MI group but did not alter the ratio in the sham group. Taken together, these data imply that there were cardiac autophagosome accumulations in the MI group, and exercise training promoted degradation of these autophagosomes.

\section{Fatty acid utilization}

Heart-type h-FABP was used as the indicator of fatty acid utilization in the heart (Figure 2A and D). Compared with the sham rabbits, the $\mathrm{Ml}$ rabbits had down-regulated cardiac expression of h-FABP. Exercise training elevated h-FABP expression in the $\mathrm{Ml}$ group, whereas it had no effect on h-FABP expression in the sham group. These result imply that MI decreased cardiac fatty acid utilization, and that exercise training could improve it.

\section{Correlation between biomarkers and left ventricular ejection fraction}

We evaluated the relationship between several biomarkers and LVEF in the present study, and found that LVEF had a significantly negative correlation with the ratio of LC3II to LC3I, but was positively correlated with h-FABP expression (Figure $3 A$ and $B$; $r=-0.509$ and 0.598 , respectively). These results suggest that left ventricular dysfunction is related more to autophagosome accumulation and less to fatty acid utilization. 

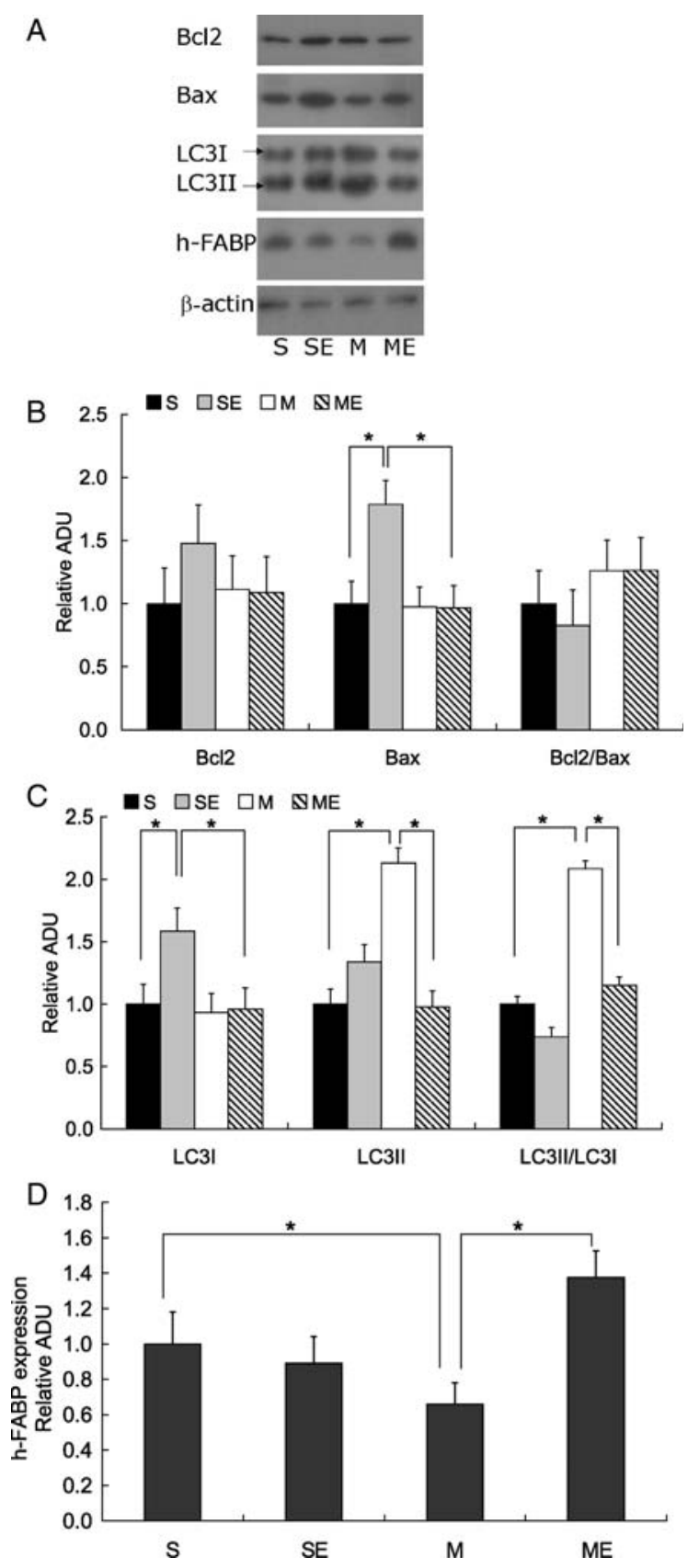

Figure 2 Effect of myocardial infarction and exercise on protein expression in the non-infarcted region of the left ventricle (counter part of the infarcted area, remote zone) by western blots (A). Bar charts showing the relative density measurements of bands obtained from all treatments. (B) Apoptotic marker (anti-apoptotic: Bcl2; pro-apoptotic: Bax). (C) Marker of autophagosome formation. (D) Fatty acid utilization in the rabbit hearts (h-FABP). Data show the means \pm SD (S and SE: $n=7 ; M$ and ME: $n=8$, respectively). S, adult rabbits with sham operation; $M$, adult rabbits with myocardial infarction for 3 months; $E$, rabbits assigned to a four-week treadmill exercise protocol at a speed of $1.0 \mathrm{~km} / \mathrm{h}$ for 30 min 5 days per week; ADU, arbitrary densitometric units $(B, C$, and $D ; *, P<0.05)$.
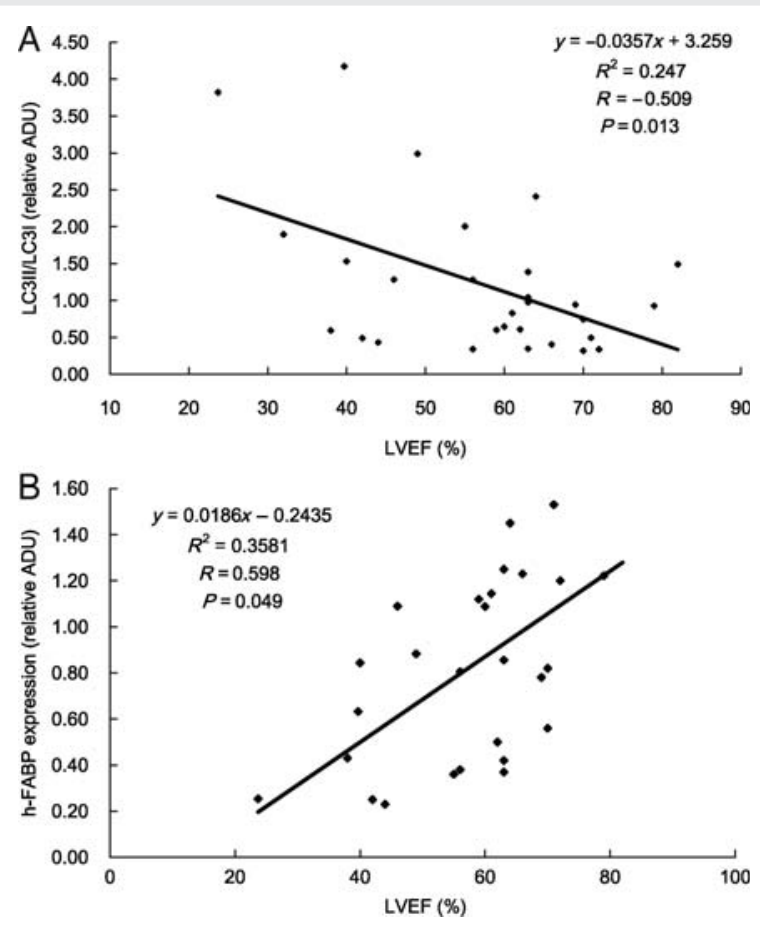

Figure 3 Correlation between left ventricular ejection fraction and other biomarkers. (A) Correlation with autophagosome formation (the ratio of LC3II to LC3I) in the rabbit hearts. (B) Correlation with fatty acid utilization (h-FABP). ADU, arbitrary densitometric units.

\section{Discussion}

The major findings in the present study are that MI caused LV dysfunction, increased lipid peroxidation, decreased autophagic function, and lower fatty acid utilization in the LV. Exercise training increased antioxidant capacity, decreased nitrosative stress, and promoted autophagic function in the sham rabbits. However, exercise training had different effects on post-MI rabbits. It improved LV function, and interestingly, it increased autophagic function and fatty acid utilization which were decreased by MI. However, exercise training did not alter the antioxidant capacity or nitrosative stress in the Ml rabbits.

In accordance with our findings, exercise significantly improved LV function in post-infarction hearts. Giannuzzi et al. ${ }^{28}$ have reported concordant results, showing that a 6-month exercise programme improved LVEF in heart failure patients. Moreover, a recent meta-analysis demonstrated that aerobic training significantly improved LVEF and decreased both end-diastolic volume and end-systolic volume. ${ }^{29}$ However, another study showed that exercise induced LV dilation and wall thinning following $\mathrm{MI}^{30}$ Currently, MRI is the most precise method for assessment of the remodelling process. Data from our previous study using MRI showed that exercise training in patients with reduced left ventricular function following $\mathrm{Ml}$ did not cause further myocardial damage such as wall thinning, infarct expansion or an increase in ventricular volume. ${ }^{31}$ In the current study, although an increase 
in the LV end-diastolic volume and a decrease in the LV endsystolic volume were noted in the exercise trained rabbits, statistical analysis did not find any significant differences in LV geometry.

It is clear that $\mathrm{Ml}$ induces oxidative stress and concordant results were seen in this study such as high TBARS in the infarcted heart. Unexpectedly, we found that the 3-NT level was significantly decreased in the infarcted hearts, and that exercise slightly increased the 3-NT level in the infarcted heart. Nitric oxide (NO) is cardioprotective via its inhibition of I/R injury, suppression of inflammation, and prevention of LV remodelling. ${ }^{32}$ Some NO metabolites, such as nitrite and nitrate, have vasculoprotective properties; whereas peroxynitrite, an oxidant metabolite of $\mathrm{NO}$, reacts with tyrosine in proteins to form 3-NT and aggravates myocardial damage in I/R injury and in chronic heart failure. ${ }^{32,33} \mathrm{Com}-$ pared with healthy subjects, chronic heart failure patients have a lower basal nitrite concentration and $\mathrm{NO}$ production, as well as a lower enhancement of exercise-induced $\mathrm{NO}$ production. ${ }^{34,35}$ Therefore, we speculated that the lower NO production in the failing heart might mean that there was less $\mathrm{NO}$ available for peroxynitrite formation resulting in less 3-NT generation. In addition, it has been reported that 3-NT generation is greater in the border zone than in the remote zone of infarcted mice hearts. ${ }^{36}$ This might explain the reduced 3-NT level in the remote LV in the $\mathrm{Ml}$ group in the present study. In addition to the change in 3-NT level, it is important to identify what kinds of proteins are modified to affect cardiac function. Lokuta et al. ${ }^{37}$ found that increased nitration of sarcoplasmic reticulum $\mathrm{Ca}^{2+}$-ATPase (SERCA2a) is seen in the idiopathic dilated cardiomyopathy (DCM) heart, which contributes to the $\mathrm{Ca}$ pump failure and hence heart failure in DCM. Therefore, we need to clarify the kind of 3-NT in proteins and to elucidate their effects, in future experiments.

The present study showed that exercise increased antioxidant capacity in the sham heart, but not in the MI heart despite an improvement in cardiac function. Accordingly, this suggests that other factors may contribute to this improvement. In the healthy animal model, exercise training increases myocardial oxygen consumption and it also increases the activity of antioxidant enzymes, thus maintaining a low oxidative status in the heart. ${ }^{11,38}$ A similar pattern was found in this study with increased antioxidant capacity in the sham heart following exercise training. Similarly, exercise increases the antioxidant enzyme activity in the skeletal muscle of heart failure patients. ${ }^{33}$ However, we found that exercise had no effect on total antioxidant capacity or levels of reduced GSH in the MI heart, but interestingly exercise did not worsen the cardiac oxidative status despite high myocardial oxygen consumption, implying that there might be another antioxidant supply from outside of the heart, such as reduced GSH via the circulation. The major source of plasma GSH is the liver; and after its synthesis, GSH is transported to supply other tissues. ${ }^{39}$ A high blood reduced GSH concentration has been reported in chronic heart failure patients compared with healthy subjects. ${ }^{40}$ Therefore, we speculate that plasma GSH was targeted to the MI heart to compensate for the oxidative stress, and thus no further oxidative damage was caused in the $\mathrm{Ml}$ heart. This might explain why there was no change in reduced GSH levels among the treatment groups in this study.
Autophagy is a major catabolic pathway by which mammalian cells degrade and recycle macromolecules and organelles. ${ }^{17,41}$ It plays a critical role in removing protein aggregates, as well as damaged or excess organelles, in maintaining intracellular homeostasis and in keeping the cell healthy. In the heart, autophagy occurs at low levels to maintain cellular homeostasis under normal conditions, and increased autophagy can be seen in conditions of starvation, I/R, and heart failure. ${ }^{15-17}$ Impairment in both formation and downstream lysosomal degradation of autophagosomes triggers mitochondrial and lysosomal pro-apoptotic pathways, and therefore culminates in cardiomyocyte death. ${ }^{15,17}$ There is increasing evidence showing that exercise activates autophagy in muscle. ${ }^{19-21}$ Resistance exercise causes a significant increase in protein degradation activities, such as lysosomal hydrolyase and Vps34, ${ }^{19,20}$ and increases the content of myocardial protein synthesis, ${ }^{20,21}$ thus stimulating protein turnover. In this study, exercise training caused an increase in LC3I and maintained a similar LC3II expression in the sham group; although in the $\mathrm{Ml}$ group it decreased LC3II expression and caused a lower ratio of LC3II to LC3I. Therefore, we hypothesize that in the MI rabbits exercise training increased protein turnover, promoted the recycling of autophagosomes, and then enhanced the autophagic degradation. Additionally, autophagosome formation was negatively correlated with cardiac function in this study (Figure 3A), implying that increasing autophagosome degradation by exercise training correlates with the improvement in cardiac function. Taken together, exercise training induced cardioprotection even partially through increasing autophagic function in the LV of the failing hearts.

Exercise has been demonstrated to induce metabolic rates and increase lipid utilization as an energy source by elevating total muscle plasma FABP content and inducing the whole body capacity for fat oxidation in healthy and post-MI subjects. ${ }^{42,43}$ In the present study, high level of TBARS and increased expression of h-FABP in the LV due to exercise training were observed, and thus we speculate that exercise training elevated myocardial lipid uptake and exposure of fatty acids to a hyper-oxygen environment might have caused the increased lipid peroxidation observed in this study. Additionally, the positive correlation between h-FABP expression and LVEF suggests that this might be involved in the improvement in cardiac function (Figure 3B).

Some experiments which were important for verifying exact mechanisms were limited in this study. Autophagy involves the delivery of the autophagosomes and their contents to lysosomes that contain the degradative enzymes needed to complete the catabolic processes of autophagy. Therefore, the reduced LC3II/ LC3I may reflect either decreased autophagosome formation, or increased turnover of autophagosomes due to activation in the degradation pathway, or a combination of the two. The best way to distinguish between 'decreased autophagosome formation and increased turnover of autophagosomes' is to measure the kinetics of autophagic flux. ${ }^{15}$ Without the kinetics of autophagic flux, less evidence could be obtained to elucidate the exact mechanism. The exploration of fatty acid utilization had similar limitations. High cardiac expression of h-FABP implies an increase in fatty acid uptake, but a better measure is the evaluation of real-time fatty acid uptake kinetics. ${ }^{44}$ The available method uses a 
fluorescently labelled fatty acid agent which is still not suitable for our animal model; this therefore limits the findings of this study.

To conclude, exercise training improved cardiac function in a post-Ml rabbit model. Exercise training promoted autophagic function and increased fatty acid utilization as well, and these factors may have contributed to the improvement in cardiac function. Exercise did not improve antioxidant capacity or oxidative stress in the Ml rabbits.

\section{Acknowledgement}

The authors are grateful to Miss Luoisa Pao and Miss Mai-Jun Lai for technical assistance.

\section{Funding}

This work was supported by Research Grants DOH97-TD-B-111-001, NTUH.97-S808 and NSC 98-2314-B-002-111. The study was sponsored, conducted, and analysed by the National Science Council, National Center of Excellence for General Clinical Trial and Research at the National Taiwan University Hospital.

Conflict of interest: none declared.

\section{References}

1. Chen CY, Lee BC, Hsu HC, Lin HJ, Chao CL, Lin YH, Ho YL, Chen MF. A proteomic study of the effects of ramipril on post-infarction left ventricular remodelling in the rabbit. Eur J Heart Fail 2008;10:740-748.

2. Leosco D, Rengo G, laccarino G, Golino L, Marchese M, Fortunato F, Zincarelli C, Sanzari E, Ciccarelli M, Galasso G, Altobelli GG, Conti V, Matrone G, Cimini V, Ferrara N, Filippelli A, Koch WJ, Rengo F. Exercise promotes angiogenesis and improves beta-adrenergic receptor signalling in the post-ischaemic failing rat heart. Cardiovasc Res 2008;78:385-394.

3. Nishiyama Y, Minohara M, Ohe M, Hirai Y, Katoh A, Miyamoto T, Iwami G, Nakata M, Koga Y. Effect of physical training on insulin resistance in patients with chronic heart failure. Circ J 2006;70:864-867.

4. Powers SK, Quindry JC, Kavazis AN. Exercise-induced cardioprotection against myocardial ischemia-reperfusion injury. Free Radic Biol Med 2008;44:193-201.

5. Gielen S, Adams V, Linke A, Erbs S, Mobius-Winkler S, Schubert A, Schuler G, Hambrecht R. Exercise training in chronic heart failure: correlation between reduced local inflammation and improved oxidative capacity in the skeletal muscle. Eur J Cardiovasc Prev Rehabil 2005;12:393-400.

6. Lawler JM, Kwak HB, Song W, Parker JL. Exercise training reverses downregulation of HSP70 and antioxidant enzymes in porcine skeletal muscle after chronic coronary artery occlusion. Am J Physiol Regul Integr Comp Physiol 2006;291: R1756-R1763.

7. Nunes RB, Tonetto M, Machado N, Chazan M, Heck TG, Veiga AB, Dall'Ago P. Physical exercise improves plasmatic levels of IL-10, left ventricular end-diastolic pressure, and muscle lipid peroxidation in chronic heart failure rats. J Appl Physiol 2008; 104:1641-1647.

8. Pullen PR, Nagamia SH, Mehta PK, Thompson WR, Benardot D, Hammoud R, Parrott JM, Sola S, Khan BV. Effects of yoga on inflammation and exercise capacity in patients with chronic heart failure. J Card Fail 2008;14:407-413.

9. Abunasra HJ, Smolenski RT, Morrison K, Yap J, Sheppard MN, O’Brien T, Suzuki K, Jayakumar J, Yacoub $\mathrm{MH}$. Efficacy of adenoviral gene transfer with manganese superoxide dismutase and endothelial nitric oxide synthase in reducing ischemia and reperfusion injury. Eur J Cardiothorac Surg 2001;20:153-158.

10. Dabkowski ER, Williamson CL, Hollander JM. Mitochondria-specific transgenic overexpression of phospholipid hydroperoxide glutathione peroxidase (GPx4) attenuates ischemia/reperfusion-associated cardiac dysfunction. Free Radic Biol Med 2008;45:855-865.

11. Duncker DJ, Stubenitsky R, Verdouw PD. Role of adenosine in the regulation of coronary blood flow in swine at rest and during treadmill exercise. Am J Physiol 1998;275: $\mathrm{H} 1663-\mathrm{H} 1672$.

12. Farias M 3rd, Gorman MW, Savage MV, Feigl EO. Plasma ATP during exercise: possible role in regulation of coronary blood flow. Am J Physiol Heart Circ Physiol 2005;288:H1586-H1590.

13. Kunapuli S, Rosanio S, Schwarz ER. 'How do cardiomyocytes die?' apoptosis and autophagic cell death in cardiac myocytes. J Card Fail 2006;12:381-391.

14. Buja LM, Vela D. Cardiomyocyte death and renewal in the normal and diseased heart. Cardiovasc Pathol 2008;17:349-374.
15. Hamacher-Brady A, Brady NR, Gottlieb RA. Enhancing macroautophagy protects against ischemia/reperfusion injury in cardiac myocytes. J Biol Chem 2006;281: $29776-29787$.

16. Nishida K, Yamaguchi O, Otsu K. Crosstalk between autophagy and apoptosis in heart disease. Circ Res 2008;103:343-351.

17. Terman A, Gustafsson B, Brunk UT. Autophagy, organelles and ageing. J Pathol 2007;211:134-143.

18. Kabeya Y, Mizushima N, Yamamoto A, Oshitani-Okamoto S, Ohsumi $Y$, Yoshimori T. LC3, GABARAP and GATE16 localize to autophagosomal membrane depending on form-II formation. J Cell Sci 2004;117:2805-2812.

19. Ilback NG, Friman G, Squibb RL, Johnson AJ, Balentine DA, Beisel WR. The effect of exercise and fasting on the myocardial protein and lipid metabolism in experimental bacterial myocarditis. Acta Pathol Microbiol Immunol Scand A 1984;92: 195-204.

20. Mackenzie MG, Hamilton DL, Murray JT, Baar K. mVps34 is activated by an acute bout of resistance exercise. Biochem Soc Trans 2007;35:1314-1316.

21. Miyazaki M, Esser KA. Cellular mechanisms regulating protein synthesis and skeletal muscle hypertrophy in animals. J Appl Physiol 2009;106:1367-1373.

22. Chen MF, Hsu HC, Lee YT. Effects of fish oil supplementation on the changes in myocardial cyclic adenosine monophosphate, inositol 1,4,5-triphosphate and mitochondrial calcium levels during acute coronary occlusion-reperfusion in cholesterol-fed rabbits. Int J Cardiol 1994;46:23-31.

23. Yang AL, Jen CJ, Chen HI. Effects of high-cholesterol diet and parallel exercise training on the vascular function of rabbit aortas: a time course study. J Appl Physiol 2003;95:1194-1200.

24. Lee BC, Hsu HC, Tseng WY, Chen CY, Lin HJ, Ho YL, Su MJ, Chen MF. Cell therapy generates a favourable chemokine gradient for stem cell recruitment into the infarcted heart in rabbits. Eur J Heart Fail 2009;11:238-245.

25. Borke WB, Munkeby BH, Halvorsen B, Bjornland K, Tunheim SH, Borge GI, Thaulow E, Saugstad OD. Increased myocardial matrix metalloproteinases in hypoxic newborn pigs during resuscitation: effects of oxygen and carbon dioxide. Eur J Clin Invest 2004;34:459-466.

26. Chen MF, Hsu HC, Lee YT. Short-term treatment with low-dose pravastatin attenuates oxidative susceptibility of low-density lipoprotein in hypercholesterolemic patients. Cardiovasc Drugs Ther 1997;11:787-793.

27. Mikkelsen RB, Wardman P. Biological chemistry of reactive oxygen and nitrogen and radiation-induced signal transduction mechanisms. Oncogene 2003;22: 5734-5754.

28. Giannuzzi P, Temporelli PL, Corra U, Tavazzi L. Antiremodeling effect of longterm exercise training in patients with stable chronic heart failure: results of the Exercise in Left Ventricular Dysfunction and Chronic Heart Failure (ELVD-CHF) Trial. Circulation 2003;108:554-559.

29. Haykowsky MJ, Liang Y, Pechter D, Jones LW, McAlister FA, Clark AM. A meta-analysis of the effect of exercise training on left ventricular remodeling in heart failure patients: the benefit depends on the type of training performed. J Am Coll Cardiol 2007;49:2329-2336.

30. McKelvie RS, Teo KK, McCartney N, Humen D, Montague T, Yusuf S. Effects of exercise training in patients with congestive heart failure: a critical review. J Am Coll Cardiol 1995;25:789-796.

31. Lee BC, Chen SY, Hsu HC, Su MY, Wu YW, Chien KL, Tseng WY, Chen MF, Lee YT. Effect of cardiac rehabilitation on myocardial perfusion reserve in postinfarction patients. Am J Cardiol 2008;101:1395-1402.

32. Rastaldo R, Pagliaro P, Cappello S, Penna C, Mancardi D, Westerhof N, Losano G. Nitric oxide and cardiac function. Life Sci 2007;81:779-793.

33. Linke A, Adams V, Schulze PC, Erbs S, Gielen S, Fiehn E, Mobius-Winkler S, Schubert A, Schuler G, Hambrecht R. Antioxidative effects of exercise training in patients with chronic heart failure: increase in radical scavenger enzyme activity in skeletal muscle. Circulation 2005;111:1763-1770.

34. Adachi H, Nguyen PH, Belardinelli R, Hunter D, Jung T, Wasserman K. Nitric oxide production during exercise in chronic heart failure. Am Heart J 1997;134:196-202.

35. Niebauer J, Clark AL, Webb-Peploe KM, Boger R, Coats AJ. Home-based exercise training modulates pro-oxidant substrates in patients with chronic heart failure. Eur J Heart Fail 2005; 7:183-188.

36. van Deel ED, Lu Z, Xu X, Zhu G, Hu X, Oury TD, Bache RJ, Duncker DJ, Chen Y. Extracellular superoxide dismutase protects the heart against oxidative stress and hypertrophy after myocardial infarction. Free Radic Biol Med 2008;44:1305-1313.

37. Lokuta AJ, Maertz NA, Meethal SV, Potter KT, Kamp TJ, Valdivia HH, Haworth RA. Increased nitration of sarcoplasmic reticulum $\mathrm{Ca}^{2+}$-ATPase in human heart failure. Circulation 2005;111:988-995.

38. Husain K, Hazelrigg SR. Oxidative injury due to chronic nitric oxide synthase inhibition in rat: effect of regular exercise on the heart. Biochim Biophys Acta 2002; 1587:75-82.

39. Adams JD Jr, Lauterburg BH, Mitchell JR. Plasma glutathione and glutathione disulfide in the rat: regulation and response to oxidative stress. J Pharmacol Exp Ther 1983;227:749-754. 
40. Campolo J, De Maria R, Caruso R, Accinni R, Turazza F, Parolini M, Roubina E, De Chiara B, Cighetti G, Frigerio M, Vitali E, Parodi O. Blood glutathione as independent marker of lipid peroxidation in heart failure. Int J Cardiol 2007;117:45-50.

41. Nakai A, Yamaguchi O, Takeda T, Higuchi Y, Hikoso S, Taniike M, Omiya S, Mizote I, Matsumura Y, Asahi M, Nishida K, Hori M, Mizushima N, Otsu K. The role of autophagy in cardiomyocytes in the basal state and in response to hemodynamic stress. Nat Med 2007;13:619-624.

42. Burelle Y, Wambolt RB, Grist M, Parsons HL, Chow JC, Antler C, Bonen A, Keller A, Dunaway GA, Popov KM, Hochachka PW, Allard MF. Regular exercise is associated with a protective metabolic phenotype in the rat heart. Am J Physiol Heart Circ Physiol 2004;287:H1055-H1063.

43. Talanian JL, Galloway SD, Heigenhauser GJ, Bonen A, Spriet LL. Two weeks of high-intensity aerobic interval training increases the capacity for fat oxidation during exercise in women. J Appl Physiol 2007;102: $1439-1447$.

44. Liao J, Sportsman R, Harris J, Stahl A. Real-time quantification of fatty acid uptake using a novel fluorescence assay. J Lipid Res 2005;46: 597-602. 